Int. J. Dev. Biol. 48: 975-980 (2004)

doi: $10.1387 / \mathrm{ijdb} .041867 \mathrm{pt}$

\title{
A newt's eye view of lens regeneration
}

\author{
PANAGIOTIS A. TSONIS*,1, MAYUR MADHAVAN², EMILY E. TANCOUS ${ }^{2}$ and KATIA DEL RIO-TSONIS 2 \\ ${ }^{1}$ Department of Biology, University of Dayton, Dayton, OH, USA and ${ }^{2}$ Department of Zoology, Miami University, Oxford, OH, USA
}

\begin{abstract}
In this paper we describe the basic process of lens regeneration in adult newt and we pinpoint several issues in order to obtain a comprehensive understanding of this ability, which is restricted to only a few salamanders. The process is characterized by dynamic changes in the organization of the extracellular matrix in the eye, re-entering of the cell cycle and dedifferentiation of the dorsal iris pigment epithelial cells. The ability of the dorsal iris to contribute to lens regeneration is discussed in light of iris-specific gene expression as well as in relation to factors present in the eye.
\end{abstract}

KEY WORDS: lens, regeneration, newt, transdifferentiation, iris

\section{A general background of lens regeneration}

Regeneration of the lens in adult newts was observed first by Collucci in 1891 and independently by Wolff in 1895, after whom the process is often called Wolffian regeneration. A few species of fish and premetamorphic frogs can also regenerate the lens. In the fish and newts the regenerating lens is derived from the pigment epithelial cells (PECs) of the dorsal iris, whereas in the frog it is derived from the cornea (Sato, 1961; Freeman, 1963). Subsequent work over several decades provided very important insights about the process and its restriction to only some adult salamanders. First, not all salamanders are capable of lens regeneration (Stone, 1967). The axolotl, which can regenerate its limbs and tail very well, is unable to regenerate the lens and this mystery might unveil crucial mechanisms (see below). Second, the PECs must re-enter the cell cycle and de-differentiate for regeneration (Eguchi and Shingai, 1971; Reyer, 1977; Yamada, 1977). Then these dedifferentiated cells can differentiate to lens cells. This process has been called lens transdifferentiation and it was clearly shown by Eguchi and colleagues in several studies including cell culture (Eguchi 1988). The ability of the dorsal and not the ventral PECs to transdifferentiate in vivo is very important, especially in relation to the fact that ventral PECs are able to transdifferentiate to lens after prolonged culturing (Eguchi, 1988). Indeed, newt PECs from the ventral iris as well as retinal pigmented epithelium (RPE) cells from many different vertebrates are capable of ttransdifferentiation to lentoids after cultured under certain condition. This is true for even aged human PECs (reviewed in Eguchi, 1988; Tsonis et al., 2001). Much work, especially using chick RPE has led to identification of factors that seem to mediate this ability of pigment epithelial cells in culture. The most notable of all are FGF, hyaluronidase, phenylthiourea and vitamin C (Eguchi, 1988; Kosaka et al., 1999).
So it seems that the ability for transdifferentiation is present in all PECs, but in vivo it can only be retained by the dorsal iris PECs of the adult newt. This calls for extensive search for factors restricted to the dorsal or ventral iris specifically and also for comparative studies with the axolotl. Thus, the processes involved in lens regeneration have become primary paradigms in the study of cell plasticity, cell determination, aging and reprogramming (Tsonis, 2000; Del Rio-Tsonis and Tsonis, 2003; Tsonis and Del Rio-Tsonis, 2004). In this paper we will address all these issues and outline research directions that most likely would provide answers to such a remarkable biological phenomenon.

\section{The process of lens regeneration}

\section{PECs re-enter the cell cycle}

It is conceivable that this is a necessary step. PECs must proliferate to create the lens vesicle and the subsequent lens. However, studies in the past have clearly indicated that the ventral iris PECs re-enter the cell cycle as well, eventhough, seemingly at a lower rate. These previous studies have shown that proliferation ensues at about 4 days post-lentectomy and that at that time both dorsal and ventral PECs show comparable levels of cell proliferation (Eguchi and Shingai, 1971; Reyer, 1977; Yamada, 1977). Following that period, proliferation rates are higher in dorsal PECs, but obviously this must correlate with the process of lens regeneration, which has been initiated from the dorsal iris. In relation to this, expression of a hyperphosphorylated form of Retinoblastoma $(\mathrm{Rb})$ has been found in the PECs of both dorsal and ventral iris (Thitoff et al., 2003). Rb is a paramount player in cell cycle regulation and when hyperphosphorylated is inactive and leads to

Abbreviations used in this paper: $\mathrm{PEC}$, pigment epithelial cell; Rb, retinoblastoma; RPE, retinal pigmented epithelium.

*Address correspondence to: Dr. Panagiotis Tsonis. Department of Biology, University of Dayton, OH, USA. Fax: +1-937-229-2021.

e-mail: Panagiotis.Tsonis@notes.udayton.edu 

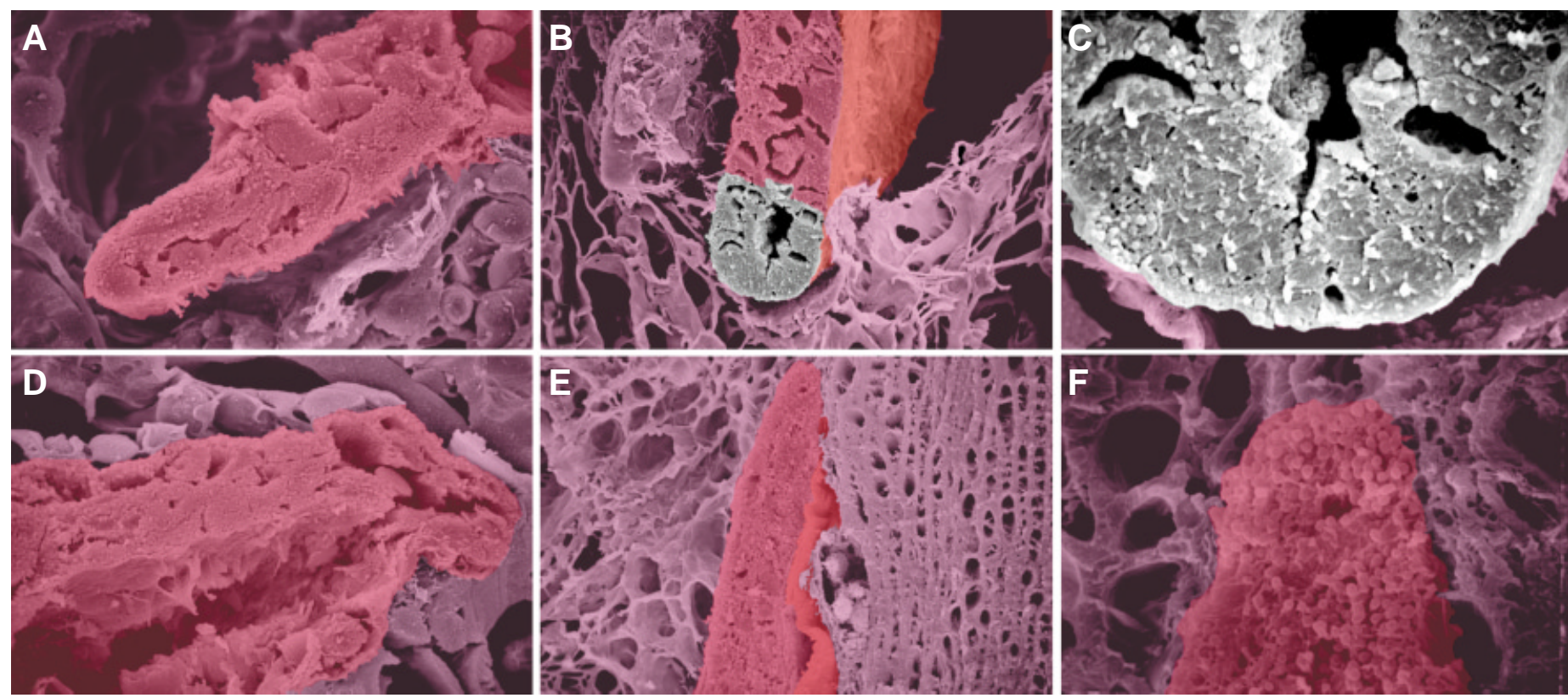

Fig. 1. Early stages of lens regeneration as depicted by SEM and pseudocoloring. (A) Dorsal iris 5 days post-lentectomy. Note that the iris is decondensing, X1,000. (B) Dorsal iris, 10 days post-lentectomy. Note that the tip of the iris has dedifferentiated and produced an early lens vesicle (gray). Colored orange is the stroma and light pink is the extracellulat matrix, X750. (C) This is a higher magnification of $B$ to show that the dedifferentiated cells lack of the globular structures that contain pigments, X3,500. (D) Ventral iris 5 days post-lentectomy. Note that the ventral iris is also decondensed, X1,000. (E) Ventral iris 10 days post-lentectomy. No dedifferentiation of PECS, X750. (F) Higher magnification of E indicating the characteristic structure of PECs with the pigment granules, X3,500. All the figures are from sections observed with a SEM.

dissociation from E2F, which then allows re-entering of the cell cycle (Rampalli et al., 1998). However, treatment with a CDK inhibitor (which is involved in $\mathrm{Rb}$ regulation and inhibits proliferation) does not completely inhibit transdifferentiation (Tsonis et al., 2004a). Also, when ventral cells are cultured for about two weeks and then implanted as aggregates in the eye cavity they can never transdifferentiate to lens, eventhough they undergo considerable proliferation (Ito etal., 1999; our observations). In different studies it has been demonstrated that thrombin activation is predominant in the dorsal iris and that its inhibition results in loss of proliferating cells (Imokawa and Brockes, 2003). Nevertheless, PECs from both dorsal and ventral iris are responsive to thrombin when stimulated in vitro (Simon and Brockes, 2002). This might imply that while proliferation is necessary to prepare cells for the process of regeneration it might not be sufficient for the subsequent transdifferentiation, and, thus, events other than the ones involved in cell cycle re-entering are decisive for transdifferentiation and lens regeneration.

\section{Transdifferentiation}

The histological events leading to dedifferentiation have been well studied at the microscopic level. Soon after lentectomy the pigment epithelial cells dedifferentiate by shedding their pigment. Macrophages recruited to the site mediate such a process. Four days post-lentectomy (a time frame that coincides with the onset of cell proliferation) the iris is decondensing and the PECs begin to elongate and at about 8-10 days these cells become columnar and depigmented (Fig. 1). The first depigmented cells are obvious at the tip of the dorsal iris (Fig. 1). At about 10 days a lens vesicle has been formed, but no crystallin expression is yet apparent.
Many of the genes that are known to be expressed and involved in lens development, such as pax-6, FGFs, FGF receptors and prox-1 are expressed in the early lens vesicle. We can confidently say that the genetic program, which is involved in lens development, is recruited during lens regeneration as well. This makes sense since the same tissue is produced in both processes. However, caution should be exercised in equating these two events especially as far as the induction signals are concerned because of the lack of adequate information at the moment. After the vesicle is formed the events of lens differentiation are initiated. At the posterior edge of the vesicle elongation of the cells ensues with concomitant induction of crystallin synthesis (Fig. 2A). The elongated cells differentiate to lens fibers and the anterior part of the vesicle becomes the lens epithelium, which continues to contribute by proliferation and differentiation to the growing of the lens (Figs. $2,3)$. Once lens differentiation has started the process is remarkably similar to lens development (as far as the sequential appearance of the different crystallins is concerned). Indeed, studies using antibodies to crystallins or cDNA probes have conclusively shown that there is a parallel of their synthesis during developing and regenerating lens. The examined crystallins were $\alpha \mathrm{A}, \beta \mathrm{B} 1$ and $\gamma$. These crystallins appeared all at the same time at the ventral portion of the lens vesicle (Sato stage IV; nearly 10 days postlentectomy), with $\gamma$-crystallin (in contrast with the other two) being specific for the lens fibers and not the lens epithelial cells (Yamada, 1977; McDevitt and Brahma, 1981; Mizuno et al., 2002). As can be seen in Fig. 4A the lens epithelium proliferates extensively throughout. As in normal lens development, when lens epithelial cells differentiate to lens fibers, they stop dividing. At the same time events of apoptosis are also observed, but at a minimal rate 

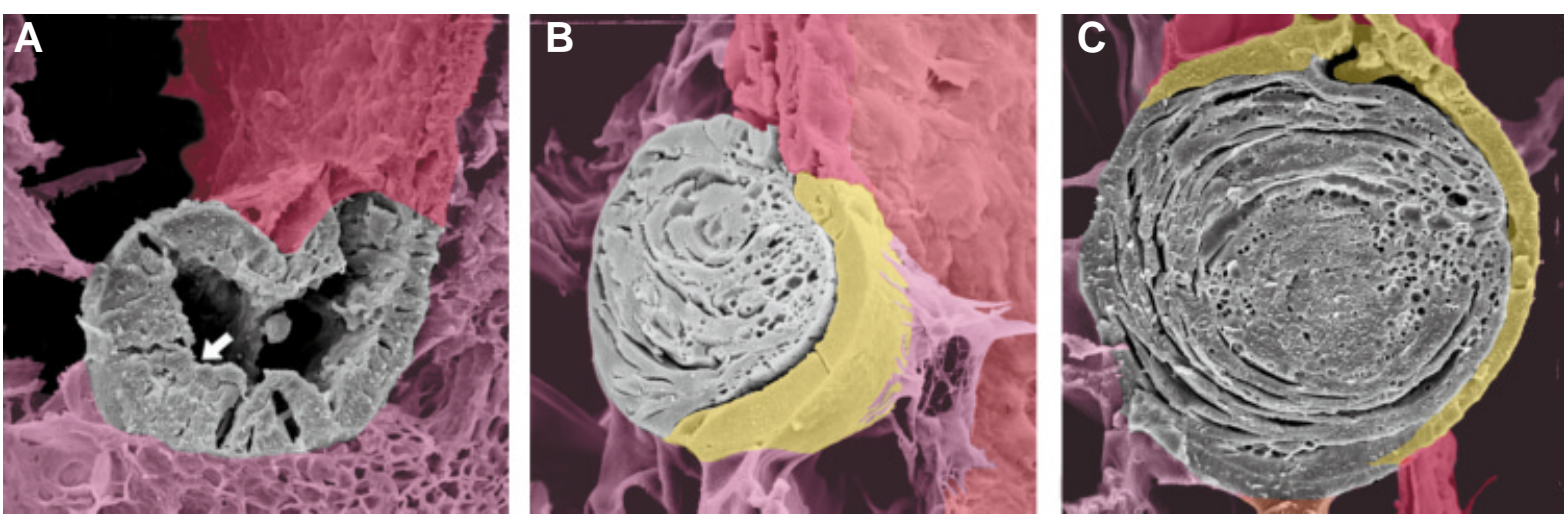

Fig. 2. (A) Regenerating lens 15 days post-lentectomy. Note that the cells in the posterior part of the vesicle (arrow) elongate as they differentiate to lens fibers. (B) Regenerating lens 20 days post-lentectomy. Note the definite formation of the lens epithelium (yellow), the differentiation of lens fibers at the equator (arrow). (C) regenerating lens 25 days post-lentectomy, depicting the more advanced stage of growth. Color code as in Fig. 1. All figures are $X 350$

(Fig. 4B; Tsonis et al., 2004a). It is interesting to note that even at stages before completion of regeneration of the lens cell death is part of the normal routine.

\section{Dynamic changes in the matrix of the eye}

The lens divides the eye cavity in two chambers. Anterior to the lens is the aqueous chamber and posterior is the vitreous chamber. As can be seen in Fig. 5A the aqueous chamber is very clear and nothing is there to disrupt the passage of the light though the lens. The situation is not the same at the vitreous chamber that separated the lens from the retina. The vitreous chamber is dominated by extracellular matrix that morphologically looks like condensed fibers and sheets. At the dorsal and ventral iris such structures are also important for the attachment of the lens. Lentectomy, however, disrupts the dissociation of the two chambers. As a result, the aqueous chamber is filled with extracellular matrix. The matrix seems less compact and is arranged in sheets that form a beehive-like structure (Fig. 5B). This matrix arrangement persists up to 15 days post-lentectomy. By 20 days we see that a compact sheet of membranes is covering the anterior part of the eye and that slowly there is a clearance in the newly established aqueous chamber (Fig. 5C). Also at this time we can observe synthesis of membranes that will eventually mediate attachment of the regenerating lens to the irises (Fig. 5C). Finally the anterior part of the lens is cleared as well. Eventhough such changes in the matrix have not been well appreciated, they might bear significance in release and/or trafficking of factors. It has been well established before that factors released from the retina are very important for the initiation of lens regeneration (Reyer, 1977). The nature of these factors is not known. However, FGFs seem to be very good candidates for such regulation. These factors are synthesized in the retina and
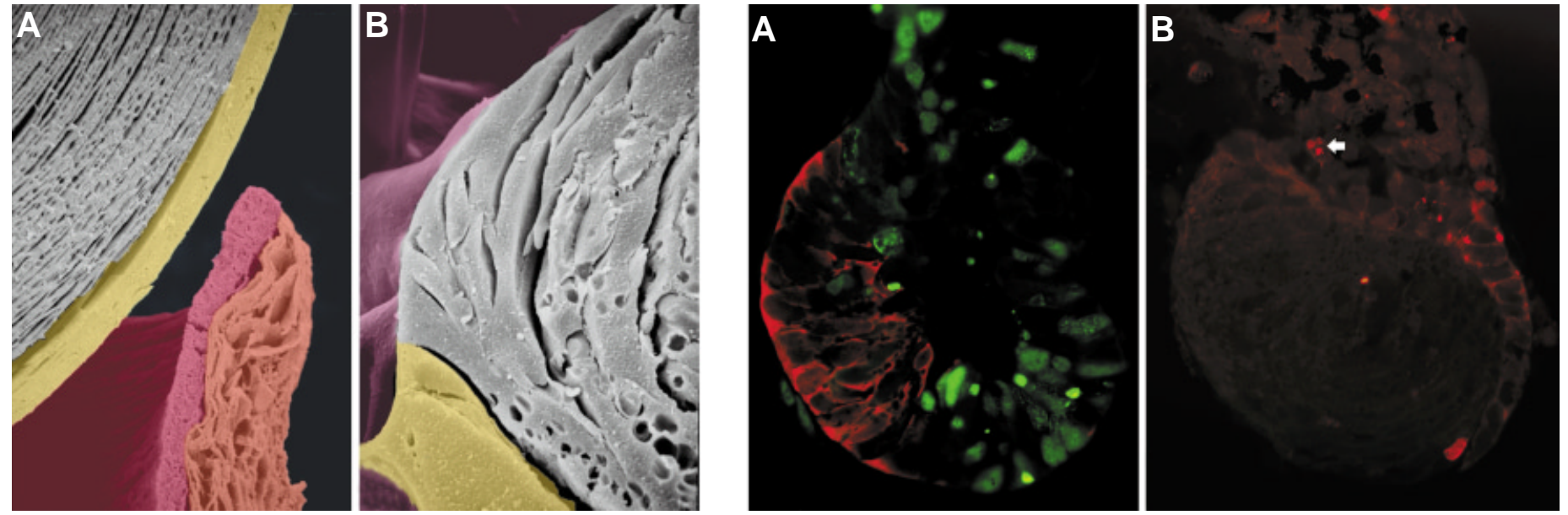

Fig. 3 (Left). Higher magnification near the bow region of intact lens (A, X750) and a regenerating lens 20 days post-lentectomy (B, X1,000). Note how compact the fibers are in the intact lens in comparison with the regenerating, owing to the continuous growth that is observed in the intact lens. Also note how compacted the iris (red) is in the intact eye and compare with the one during lens regeneration (Fig. 1A,D).

Fig. 4 (Right). Profiles of cell proliferation and crystallin synthesis (A) and apoptosis (B) during lens regeneration. (A) 15 days post-lentectomy crystallin is stained red with an antibody directed to fiber-specific $\beta$-crystallin antibody and proliferating cells are green (BrdU incorporation). Note extensive proliferation in the dorsal iris and in lens epithelium. The posterior cells are differentiating to lens fibers. (B) An apoptotic cell (arrow) in the dorsal iris and lens epithelium of a regenerating lens 20 days post-lentectomy. 

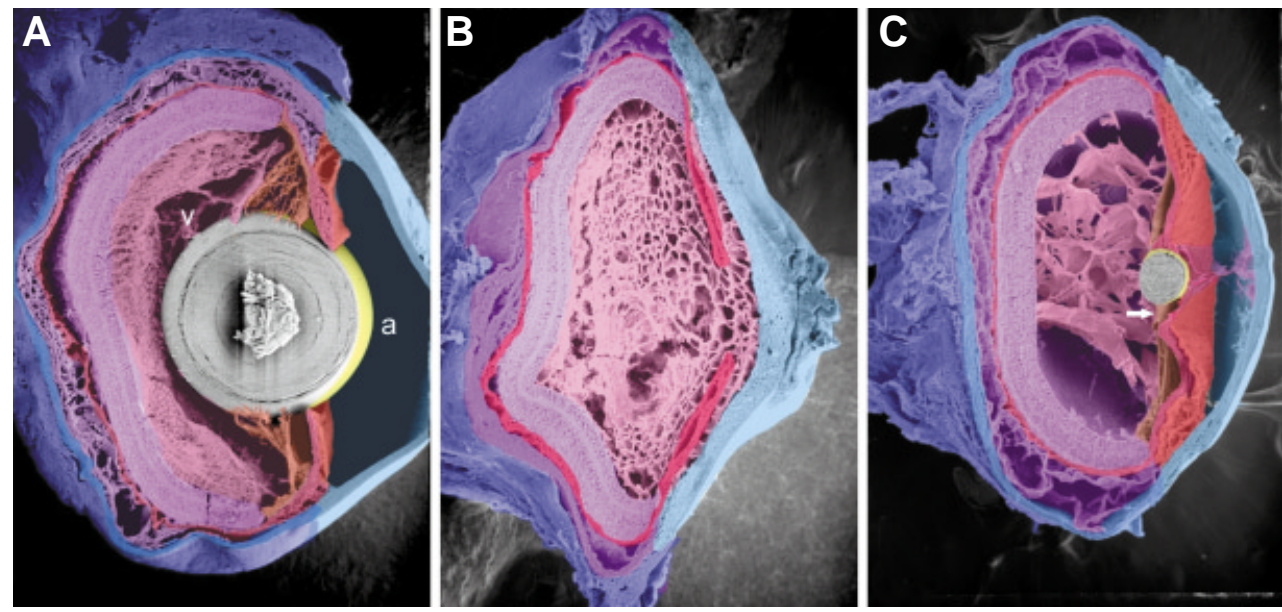

maybe there is a subsequent inhibitory event in the ventral iris. Unfortunately not much information exists about expression of key molecules in the intact dorsal and ventral iris. It is imperative to undertake a detailed analysis of gene expression in both irises before and after lentectomy. Using methods of genomics and proteomics, molecular signatures could be unveiled. This should provide valuable information about the identity issue of the dorsal iris.

Related to the above discussion the thorny question arises whether or not such a capability for lens regeneration can be assigned to presence of a single factor alone. Drawing examples from the field of eye development the answer should be more elaborate than a simple yes or no. It is true that genes such as pax-6 and six-3 have been found to induce lens formation when ectopically expressed (Halder et al., 1995; Oliver et al., 1996). This might indicate that one factor could do this, but the picture during lens regeneration, we believe, will turn

their graded presence in the vitreous and aqueous humor control the differentiation of lens fiber cells at the posterior region of the lens during development (Caruelle et al., 1989).

\section{Approaching the dorsal (or ventral) iris identity issue}

\section{Specific gene regulation along the dorsal-ventral irises}

The main challenge here is to find whether or not specific factors in either dorsal or ventral PECs are responsible for their identity. If reentering the cell cycle is not sufficient for the subsequent events of transdifferentiation, then we should expect that a unique regulatory factor (?) should be the determinant of dorsal iris identity, which will induce regeneration from that site. The hypothesis is that if such a factor is found it can then be used to engineer the ventral iris to undergo regeneration. Same scenario could be followed with the disappearance of factor(s) from the ventral iris as well.

However, to date no such a factor or events have been conclusively confined to dorsal iris. Earlier studies had suggested that certain cell surface proteoglycans and RNA are sequentially lost from the dorsal iris as dedifferentiation ensues. However, comparative studies with the ventral iris were not presented and therefore these events cannot be attributed to transdifferentiation (Zalikand Scott, 1973). Expression of other genes (important for eye or lens determination) has also been studied during the process of regeneration. Among these, pax6 , FGFR-1 and prox-1 have been shown to have a preferential expression in the dorsal iris, however, the ventral iris also expresses pax-6 and prox-1 (Del Rio-Tsonis et al., 1995, 1997, 1999; Mizuno et al., 1999). Recently, in another study, it has been shown that the Hedgehog pathway might be involved in lens regeneration, however, Shh and Ihh are expressed in both dorsal and ventral iris after lentectomy (their expression could be related to cell proliferation) (Tsonis et al., 2004b). These expression patterns might indicate that some activation could take place in the ventral iris as well and that out to be more complicated. Indeed, several factors have been identified as eye or lens-inducing factors, but it now seems that all these factors are members of a regulatory network, with multiple feedback loops and dosage-dependent effects (Goudreau et al., 2002; Zuber et al., 2003). Likewise, the same might be happening during lens regeneration. The use of mutants should provide valuable information in this area, but transgenesis or mutagenesis techniques in the newt are at present unavailable. The use of Xenopus, however, could compliment this research very well. Recent technology using the frog can target genes in the lens and knockdown screens using

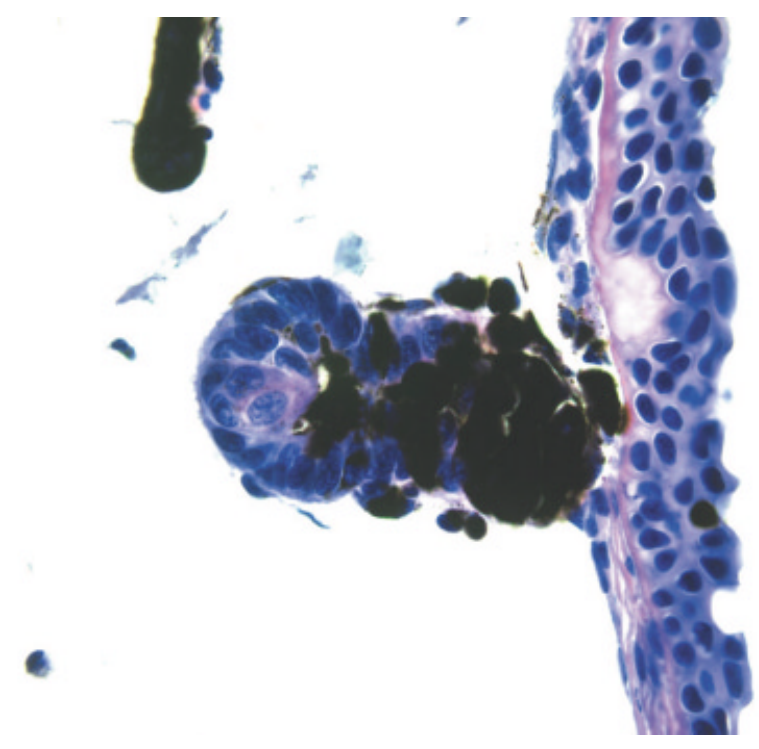

Fig. 6. Lens transdifferentiation from a piece of newt dorsal iris implanted in the axolotl eye cavity after lentectomy. 
morpholinos are now possible (Henry and Elkins, 2001; Hirsh et al., 2002; Kenwrick etal., 2004). A thorough screening in lens regeneration might identify mutants and subsequently the gene(s) involved.

\section{Is the regenerative ability of the dorsal iris intrinsic?}

Certain experiments have suggested that this indeed might be the case. This conclusion has been mainly derived from experiments involving same species or cross-species transplantation of the dorsal iris. When a piece of newt dorsal iris or aggregated PECs from the dorsal iris are transplanted into the regenerating newt limb a well differentiated lens, sometimes with impressive A-P polarity is produced (Ito et al., 1999). This means that a factor in the eye is not necessary. Alternatively, if a factor is needed, it could mean that the regenerating limb supplies this factor and that possibly similar signals are involved in two different regenerative phenomena. However, when a piece of newt dorsal iris is transplanted in the eye of an axolotl (a salamander that regenerates the limbs, but not the lens) a lens can be obtained as well! (Fig. 6) (Reyer, 1956). This could argue that a factor might not be necessary and that the ability of the dorsal iris to transdifferentiate to lens is intrinsic. It also suggests that the axolotl eye does not contain an inhibitor. Another explanation could be that the dorsal iris has a regeneration-specific regulator, but a factor is needed as well. In this case the factor could be a ligand (and the axolotl has it) but the iris has a defective or post transcriptionally modified/repressed receptor. If this is true, then the ventral iris of the newt (and the irises of other vertebrates) must have the same regulation in this putative receptor, but also to complicate things, there could be additional regulation of other factors that compete for the receptor! We would like to propose that a mutation in this receptor should be out of the question. It is hard to account for a gene that has been mutated in one area of the iris and not the other. However, a mutation in regulatory sequence could be possible. Experiments with cross-species transplantations could in fact be more informative and should be extended. For example, ventral iris PECs are known to be able to transdifferentiate to lentoids after prolonged period on culture. This might indicate that dissociation triggers some crucial events on the cell surface that could lead to transdifferentiation. Crossspecies transplantation should be undertaken with cultured cells as well. This might define a critical timing in the ability for transdifferentiation.

\section{Can the ventral iris be induced to transdifferentiate?}

Having discussed these issues we must stress that it is possible to transform the ventral iris to assume dorsal iris identity and transdifferentiate to lens. A potent carcinogen (Methyl-NitroNitrosoguanidine) has been shown to elicit regeneration of a lens from the ventral iris as well (Eguchi and Watanabe, 1973). Is this, however, a genetic or epigenetic change? In these experiments the carcinogen was applied as a crystal in one dose immediately after lentectomy and might have not affected proliferating cells to induce mutations. By the time of the onset of proliferation (4 days post-lentectomy) the carcinogens might have been metabolized. Therefore, most likely the effect of this carcinogen was either in inducing regulation of a gene that is responsible for lens transdifferentiation or the treatment resulted in epigenetic alterations in cell surface and cell to cell communication, which in turn perturbed the ventral PECs toward assuming dorsal iris properties. Indeed, Eguchi (1988) has reported preliminary studies where ventral irises treated with a monoclonal antibody (reacting to an antigen that disappears from the dorsal site after lentectomy) were able to transdifferentiate to lens. This antigen is a cell surface marker, but its identity was never revealed and subsequent studies were not pursued. However, this does show that transforming the ventral iris to dorsal is possible.

Work in our laboratories is taking the above issues into serious consideration. We strongly believe that inducing the ventral iris to transdifferentiate to lens and identifying factors that are involved will lead to a breakthrough not only in the field of regeneration, but cell fate determination, reprogramming and aging. Future directions should include revisiting older experiments with a fresh look using modern technology, especially as it relates to global and comparative gene expression. These studies are completely lacking in the field and they are much needed. Since many different tissues of the adult newt have the ability to regenerate via transdifferentiation, genomic studies might identify molecular signatures common to different regenerative processes in the newt. Such signatures can be then compared to common signatures found in stem cells (Ivanova et al., 2002; RamahloSantos et al., 2002). Eventually this will lead to understanding the molecular mechanisms of the different ways that tissues and animals employ to repair damaged tissues.

\section{Acknowledgements}

This work was supported by NIH grant to PAT and by the Miami University undergraduate research award to EET. We also thank Thomas Litzinger for help with the SEM work.

\section{References}

CARUELLE, D., GROUX-MUSCATELLI, B., GAUTRIC, A., SESTIER, C., COSCAS G., CARUELLE, J.P. and DARRITAULT, D. (1989). immunological study of acidic fibroblast growth factor ( $\alpha \mathrm{FGF}$ ) distribution in the eye. J. Cell. Biochem. 39: $117-128$

COLLUCI, V.L. (1891). Sulla rigenerazione parziale dell'occhio nei Tritoni- Istogenesi e sviluppo. Studio sperimentale. Mem. R. Acad. Sci. Ist. Bologna, Ser. 51:593629.

DEL RIO-TSONIS, K., JUNG, J.C., CHIU, I-M. and TSONIS, P.A. (1997) Conservation of fibroblast growth factor function in lens regeneration. Proc. Natt. Acad. Sci. USA 94: 13701-13706.

DEL RIO-TSONIS, K., TOMAREV, S.I. and TSONIS, P.A. (1999). Regulation of prox-1 during lens regeneration. Invest. Ophthalmol. Vis. Sci. 40: 2039-2045.

DEL RIO-TSONIS, K. and TSONIS, P.A. (2003). Eye regeneration at the molecular age. Dev Dyn. 226(2):211-224.

DEL RIO-TSONIS, K., WASHABAUGH, C.H. and TSONIS, P.A. (1995). Expression of pax-6 during urodele eye development and lens regeneration. Proc. Nat/. Acad. Sci. USA 92: 5092-5096.

EGUCHI, G. (1963). Electron microscopic studies on lens regeneration. I. Mechanisms of depigmentation of the iris. Embryologia 8: 45-62.

EGUCHI, G. (1964). Electron Microscopic studies on lens regeneration. II. Formation and growth of lens vesicle and differentiation of lens fibers. Embryologia 8: 247287

EGUCHI, G. and SHINGAI, R. (1971). cellular analysis on localization of lens forming potency in the newt iris epithelium. Develop. Growth Differ. 13:337-349.

EGUCHI, G. and WATANABE, K. (1973). Elicitation of lens formation from the ventral iris epithelium of the newt by a carcinogen, N-methyl-N'-nitro- $\mathrm{N}$ nitrosoguanidine. J. Embryol. Exp. Morphol. 30: 63-71.

EGUCHI, G., ABE, S.I. and WATANABE, K. (1974). Differentiation of lens-like structures from newt iris epithelial cells in vitro. Proc. Natl. Acad. Sci. USA. 71(12): 5052-5056. 
EGUCHI, G. (1988). Cellular and molecular background of Wolffian lens regeneration. In: Regulatory mechanisms in developmental processes. (G. Eguchi, T.S. Okada, L. Saxen, eds) Elsevier, Ireland, 147-158.

FREEMAN, G. (1963). Lens regeneration from the cornea in Xenopus laevis. J. Exp. Zool. 154: 39-66.

GoudreAU, G., PETROU, P., RENEKER, L.W., GRAW, J., LOSTER, J. and GRUSS P. (2002). Mutually regulated expression of pax- 6 and six-3 and its implications for the pax-6 haploinsufficient lens phenotype. Proc. Natl. Acad. Sci. USA 99: 87-19-8724.

HALDER, G., CALLAERTS, P. and GEHRING, W.J. (1995). Induction of ectopic eyes by targeted expression of the eyeless gene in Drosophila. Science 267: 1788-1792.

HENRY, J.J. and ELKINS MB. (2001). Cornea-lens transdifferentiation in the anuran, Xenopus tropicalis. Dev. Genes Evol. 211: 377-87.

HIRSCH, N., ZIMMERMAN, L.B., GRAY, J., CHAE, J., CURRAN, K.L., FISHER, M., OGINO, H. and GRAINGER RM. (2002). Xenopus tropicalis transgenic lines and their use in the study of embryonic induction. Dev Dyn. 225: 522-535.

IMOKAWA, Y. and BROCKES, J.P. (2003). Selective activation of thrombin is a critical determinant for vertebrate lens regeneration. Current Biol. 13: 877881.

ITO, M., HAYASHI, T., KUROIWA, A. and OKAMOTO M. (1999). Lens formation by pigmented epithelial cell reaggregate from the dorsal iris implanted into the limb blastema in the adult newt. Develop. Growth Differ. 41: 429-440.

IVANOVA, N.B., DIMOS, J.T., SCHANIEL, C., HACKNEY, J.A., MOORE, K.A. and LEMISCHKA, I.R. (2002). A stem cell molecular signature. Science 298: 601604.

KENWRICK, S., AMAYA, E. and PAPALOPULU, N. (2004). Pilot morpholino screen in Xenopus tropicalis identifies a novel gene involved in head development. Dev. Dyn. 229: 289-99.

KOSAKA, M., KODAMA, R. and EGUCHI, G. (1998). In vitro culture system for irispigmented epithelial cells for molecular analysis of transdifferentiation. Exp. Cell Res. 245: 245-251.

MCDEVITT, D.S. and BRAHMA, S.K. (1981). Ontogeny and localization of the $\alpha$, $\beta$ and $\gamma$ crystallins in newt eye lens development. Dev. Biol. 112:693-454. and

MIZUNO, N., MOCHII, M., YAMAMOTO, T.S., TAKAHASHI, T.C., EGUCHI, G. and OKADA, T.S. (1999). Pax-6 and Prox 1 expression during lens regeneration from Cynops iris and Xenopus cornea: evidence for a genetic program common to embryonic lens development. Differentiation 65(3): 141-149.

MIZUNO, N., AGATA, K., SAWADA, K., MOCHII, M. and EGUCHI, G. (2002). Expression of crystallin genes in embryonic and regenerating newt lenses. Dev. Growth Differ. 44:251-256.

OLIVER, G., LOOSLI, F., KOSTER, R., WITTBRODT, J. and GRUSS, P. (1996). Ectopic lens induction in fish in response to murine homeobox gene six-3. Mech. Develop. 60: 233-336.
RAMALHO-SANTOS, M., YOON, S., MATSUZAKI, Y., MULLIGAN, R.C. and MELTON, D.A. (2002). "Stemness": Transcriptional profiling of embryonic and adult stem cells. Science 298: 597-600.

RAMPALLI, A.M., GAO, C.Y., CHAUTHAIWALE, V.M. and ZELENKA, P.S. (1998). $\mathrm{pRb}$ and $\mathrm{p} 107$ regulate E2F activity during lens fiber cell differentiation. Oncogene 16: 399-408.

REYER, R.W. (1956). Lens regeneration from homoplastic and heteroplastic implants of the dorsal iris into the eye chamber of Triturus viridescens and Amblystoma punctatum. J. Exp. Zool. 133: 145-190.

REYER, R.W. (1977). The amphibian eye: Development and regeneration. In: Crescitelli $F$, editor. Handbook of Sensory Physiology, Volll/5: The visual System in Vertebrates. Berlin: Springer-Verlag. p. 309-390.

SATO, T. (1961). Uber die linsen-regeneration bei den cobitiden fischen Misgurnus anguillicaudatus. Embryologia 6: 251-291.

SIMON, A. and BROCKES, J.P. (2002). Thrombin activation of S-phase reentry by cultured pigmented epithelial cells of adult newts. Exp. Cell Res. 281: 101-106.

STONE, L.S. (1967). An investigation recording all salamanders which can and cannot regenerate a lens from the dorsal iris. J. Exp. Zool. 164: 87-104.

THITOFF, A.R., CALL, M.K., DEL RIO-TSONIS, K. and TSONIS, P.A. (2003). Unique expression patterns of the retinoblastoma $(\mathrm{Rb})$ gene in intact and lens regeneration-undergoing newt eyes. Anat. Record A271: 185-188.

TSONIS, P.A. (2000). Regeneration in Vertebrates. Dev. Biol. 221: 273-284.

TSONIS, P.A., JANG, W., DEL RIO-TSONIS, K. and EGUCHI, G. (2001). A unique aged retinal pigmented epithelial cell line useful for studying lens differentiation in vitro. Int. J. Dev. Biol. 45: 753-758.

TSONIS, P.A. and DEL RIO-TSONIS, K. (2004). Lens and retina regeneration: Transdifferentiation, stem cells and clinical applications. Exp. Eye Res. 78: 161172.

TSONIS, P.A., MADHAVAN, M., CALL, M.K., GAINER, S., RICE, A. and DEL RIOTSONIS, K. (2004a). Effects of a CDK inhibitor on lens regeneration. Wound Repair Reg. 12: 24-29.

TSONIS, P.A., VERGARA, M.N., SPENCE, J.R., MADHAVAN, M., KRAMER, E.L., CALL, M.K., SANTIAGO, W.G., VALLANCE, J.E., ROBBINS, D.J. and DEL RIO-TSONIS, K. (2004b). A novel role of the hedgehog pathway in lens regeneration. Dev. Biol. 267: 450-461.

WOLFF, G. (1895). Entwicklungsphysiologische Studien. I. Die Regeneration der Urodelenlinse. Wilhelm Roux Arch. Entwickl.-Mech. Org. 1: 380-390.

YAMADA, T. (1977). Control mechanisms in cell type conversion in newt lens regeneration. Monographs in Dev. Biol. 13. Karger, Basel.

ZALIK, S.E. and SCOTT, V.(1973). Sequential disappearance of cell surface components during dedifferentiation in lens regeneration. Nature New Biol. 244: 212-214.

ZUBER, M.E., GESTRI, G., VICZIAN, A.S., BARSACCHI, G. and HARRIS, W.A. (2003). Specification of the vertebrate eye by a network of eye field transcription factors. Development 130: 5155-5167. 